\title{
Smart City and Smart-Health Framework, Challenges and Opportunities
}

\author{
Majed Kamel Al-Azzam ${ }^{1}$ \\ Business Administration Department \\ Yarmouk University Irbid, Jordan
}

\author{
Malik Bader Alazzam ${ }^{2}$ \\ Software engineering Department \\ Jadara University Irbid, Jordan
}

\begin{abstract}
The new age of mobile health is accompanied with wider implementation of ubiquitous and pervasive mobile communication and computing, that in turn, has brought enormous opportunities for organizations and governments to reconsider their healthcare concept. Alongside, the global process of urbanization signifies a daunting test and attracts the expert concentration towards towns that can obtain significant high populations and service people in a human and efficient approach. The consistent need of these two trends led to evolution of the concept of smart cities plus mobile healthcare. The given article is intended to provide an overview of smart health, explained to be context-aware that is accompanied by mobile health within the smart cities. The purpose of the article is to offer a standpoint on the main fields of research and knowledge explained in the procedure of establishment of the new idea. Furthermore, the article will also focus on major opportunities and challenges that are implied by s-health and will offer a common opportunity for future research.
\end{abstract}

\section{Keywords-Smart city; challenges; opportunities; smart health}

\section{INTRODUCTION}

Electronic health (e-health) is the contribution of (ICT) implementation in the healthcare industry. This e-health concept further helps in increasing efficiency and cost reduction [1]. The e-health consolidation is followed by the widespread preference of mobile devices with abilities such as smart phones, which led to evolution of mobile health (mhealth) concept. One can understand $m$-health as delivering healthcare services using mobile communication devices [2]. $\mathrm{m}$-health offers astonishing opportunities being an addition to the already existing of e-health that are associated with the ubiquity of mobile devices, which includes immediacy, wider availability, and global monitoring capabilities [3][4]. Even when substantial innovations have been made in the field of $\mathrm{m}$ health, but the concept is still in its easy phases and is consistently developing alongside another opportunistic and promising idea of smart cities based on ICT and target to handle local concerns ranging, transportation and local economy to e-governance plus quality of life.

Local authorities, nowadays, invest hefting amounts in ICT for equipping their smart cities with all needed technological infrastructures that can foster social responsibility, support ambient intelligence, and can enhance appreciation for the environment. Hence, this indicates boundless potentials for the smart cities plus organizations. Example, Intel and IBM are partnering in a way to consolidate or merge their leadership in the given industry. They have recognized various relevant fields in which smart cities can play a crucial roles and these include energy and public utilities, public safety, education, trade and industry development, healthcare, and social services, etc. The sensors are the basis of the smart cities that offer updated information regarding distinct variables that entail humidity, temperature, pollution, concentration of allergens, traffic conditions and many more. Explored the framework as the ecological settings plus states that also help in determining behavior of an application or in which event of an application happens and is of interest to the user. These variables offered by the infrastructure of smart city are the context that can help in understanding the citizens' living environment at any time. Hence, by using this information in a proper way can help in providing patients and citizens with the healthcare services and applications with active awareness of the context (i.e., services and applications that inevitably adjust to discovered context) by making changes in the behavior of services and applications.

The prime objective of the given work is to evaluation the idea of Smart Health (s-Health) like an outcome of the natural effort or collaboration of smart cities and m-health, from the viewpoint of ICT, society, and that of individuals. The article will then focus on advantages and challenges that are explored by this new perception of health in the smart cities and analyze its practical viability.

The remaining editorial is prearranged as follows. The first segment recapitulates major and prime research field that explains a primary role in the s-health development. After this, the article will explain the idea of s-health and focus on its importance, impact, feasibility, and timeliness. Following this, the emphasis of the article will be the elaboration on the major opportunities and challenges implied by s-health. Finally, the article will be concluded by providing a summary of overall contribution of the researcher to the article and some final opinions and viewpoints about it [5].

\section{SMART CITY}

Smart city is still considered as vague concept that has not been defined strictly. Caragliu in offered a definition of the concept, which was further explained in Pérez-Martínez et al. as: these are the cities that are powerfully created on ICTs investing in social plus human capital enhancing the quality lifestyle of their citizens by promoting economic growth, wiser resource organization, engaging governance, efficient mobility, and sustainability, while they assure the security and privacy of the citizens [12]. 
The Smart cities have become a forthcoming necessity that has recently attained a great attention from both academia and industry. Private organizations such as Siemens, Intel plus IBM largely invest in smart cities. Additionally, the scientific society has also ongoing undertaking an in-depth analysis of the concept of smart cities [11].

According to the recent reports, there is an exponential increase in the pace of urbanization. At present, around 50 percent of the globe people reside in cities, and this is predictable to increase further to 70 percent by 2050 . Thus, infrastructural expansion to fulfill requirements of the large population is vital. Furthermore, infrastructures of big cities require efficiency in varied aspects that ranges from consumption of energy to allocation of resources. Hence, the only way in which cities can provide a quality life and sustainability to their people is by using "smart" communication on the gadgets through ICT to assure admission to the desired context-aware information [6].

Various cities have by now initiated working towards adopting this concept. There are four areas around the notion of sustainability that was determined by Amsterdam and these include mobility, working, public space, and living. The smart projects are conducted in these four areas for improving the city. In Amsterdam, they concentrate on reducing the emissions of $\mathrm{CO} 2$, but the researcher could also discover some examples that concentrate on facets also3. In some instances of cities that are chasing "smartness" include Toronto, Vienna, London, Paris, Copenhagen, New York, Barcelona, and Hong Kong [7][8].

The prevalent and extensive implementation of specific sensors in the smart cities offers supplement connections in the course of participatory, people-centric, as well as resourceful sensing $[8,9]$. As per the given context, a smart city turns into an enormous system of systems, which is required to offer the processed information to its local authorities and citizens. In most situations can offer personalized information that allows them using the on-request service providers for managing cities and creating the drive for corrective acts (Fig. 1).

"Smart health (s-health) explained as the provision of health services in the preference to use context-aware network and sensing infrastructure of the identified smart cities [9]."

\section{A. Smart Health}

In reference to this definition, smart health can be grouped as a subset of e-health given s-health is in relation to the ICT infrastructure of the identified a smart city. Nevertheless, there is a difference between s-health and m-health. For instance, in s-health there is a possibility that the identified fundamental communication may not be mobile or not [10] [11]. In reality, for the majority of cases it may include established fixed sensors. The identified examples that were illustrated in Fig. 2 will help in clarifying the aim of the above concepts, in reference to the subsets that are exemplified in Fig. 2.

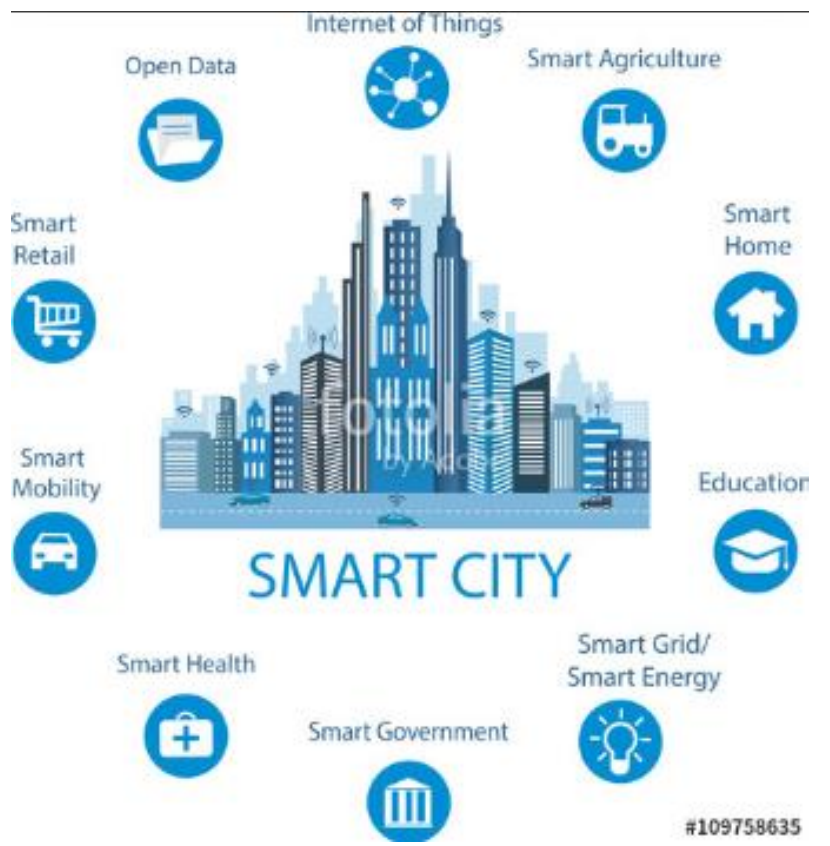

Fig. 1. Smart city components

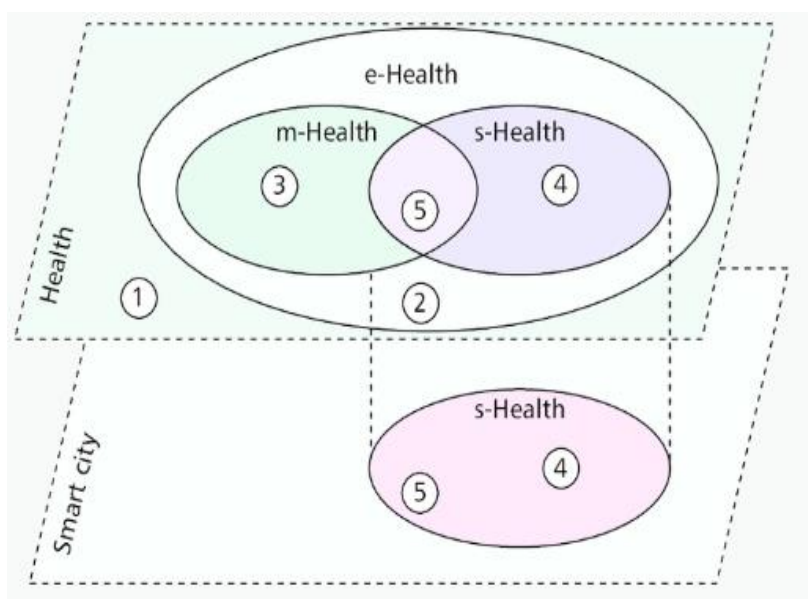

Fig. 2. Smart City and Smart Health.

1) Case 1-Classical health explained as generally activity related to health, which means a doctor that visits patients with the specified traditional tools, which do not essentially entail ICT.

2) Case 2-E-Health entails usage of databases and electronic health records (EHR) that help in saving or storing patients' medical information[1],[2],[3].

3) Case 3-M-Health. The example of this type of activity is when patient is able to check their prescriptions from their personal mobile devices for guaranteed adherence to the medications. This m-health explained as a subset of ehealth given it makes use of medical devices for accessing medical related figures. 
4) Case 4-S-Health. The patient receives information or data from an interactive pole of information for checking the level of dust, pollen, plus pollution for which individual has allergy. The information further helps patients in preventing the areas that can prove to be hazardous for their health conditions. The pole of information helps in providing the patient with information about the best route or direction that they can take to reach a destination and about the closest pharmacies from where he can purchase antihistamine pills.

5) Case 5-M-Health amplified with s- Health.This can be explained with a cyclist who is wearing a band or bracelet that has in-built accelerometers and accident is the main monitoring capability of the device. The body sensor network will help in detecting the fall of the individual and will send an notification to the city infrastructure. As soon as the system receives notification, the traffic circumstances are being evaluated, and an ambulance will be dispatched using the best selected route [4]. Additionally, it is possible to adjust the city traffic lights in a dynamic manner to minimize the time spent before the ambulance arrives at the scene [5],[6].

This shows that the prime objective of s-health is endorsement of health to a senior place within a community in a private, efficient, secure, sustainable, and distributed manner by re-utilizing the values of smart cities and $m$-health in an identified new example of pervasive fitness.

The consolidation of the new s-health concept and its definition will expand the health coverage from heath centers and adjusted homes universally in the selected smart city. The, article can also offer the ground or basis to develop new models, techniques, synergies, and interactions that will further helps in reducing the costs of health, enhancing the patients' quality of life, early illness detection, gathering invaluable research data, and the worldwide enhancement of communities [7],[8],[9].

\section{B. S-Health Vs. M-Health}

The concept of s-health can just be measured as an expansion of improvement in the notion of $\mathrm{m}$-health, which simultaneously consists of sensing abilities of the smart cities. In general, both these concepts are substantially different from one another, which in turn, justify the adoption and application of s-health concept. Even when it is evident that s-health notion cannot be confusing with the notion of smart cities, it is necessary to highlight the difference of s-health with that with m-health. The main distinctions between the two concepts include:

1) Distinctions in sources of information: The data or information that is used in the concept of m-health is generally comes from the patients. Nevertheless, the data used in shealth concept are not just completely come from patient but from independent sources such as the smart city sensing infrastructure, which is considered to be the new information source. This new source of information exceeds or is better than the m-health and hence, it rationalizes the evolution of the ideology of s-health [5],[10],[11].

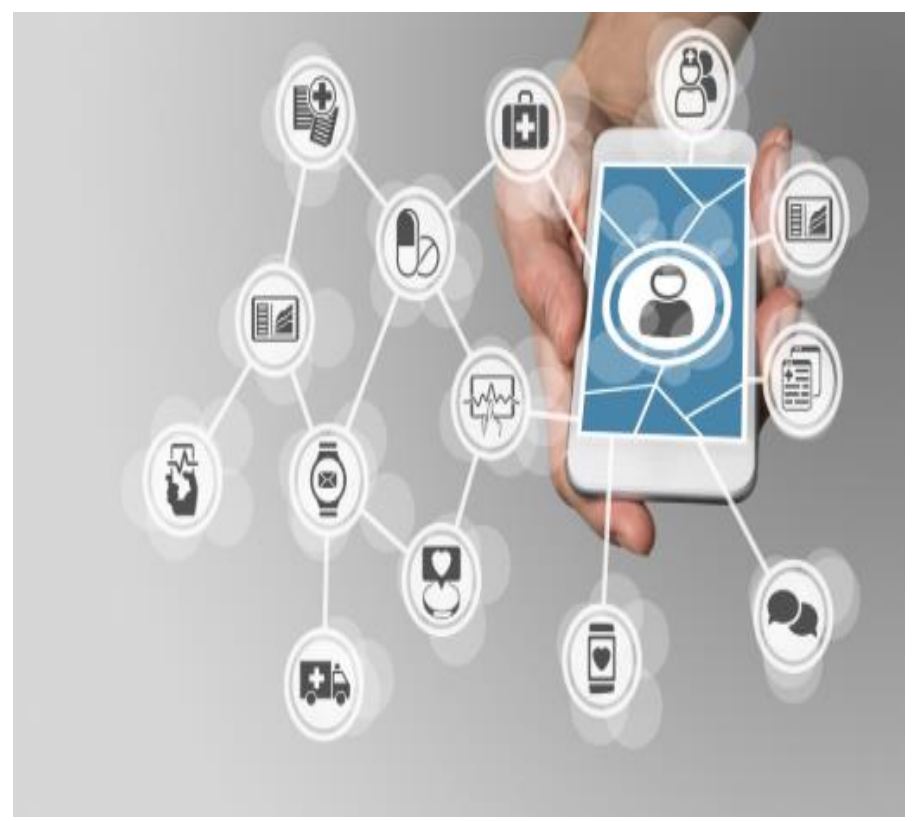

Fig. 3. M-Health.

Thus the revealed source of information exceeds m-health and explains the manifestation of the new perception of shealth [12],[4].

2) Distinctions in flow of information: $m$-Health is generally user-focused or personalized approach, whereas shealth is city-centric as well. This is proved by the face that in $\mathrm{m}$-health, information is gathered through patients and are processes and the final outcomes go back to the patients only. On the contrary, in case of s-health, which is not user-centric, the information that is obtained by the patients also changes the city's behavior, which means the concept is city-centric. As an example, if one can refer example 5 above, the fall of the cyclist in the example shows changes in the whole city's behavior and also leads to changes in the traffic lights of the city that also helps in ambulance's easiest arrival (which clearly is outside the m-health's scope).

\section{Challengesand Opportunities Challenges}

The perception of s-health consists of an enormous research that usually work in an independent manner and hence, in certain cases lead to confusions. The citizens those adopt the shealth paradigm needs certain kind of fulfillments including financial, technical, logistics, as well as psychological needs [13]. Some of the challenges that s-health is expected to conquer include:

1) Multidisciplinary research and interaction: Explored areas that are previously explained are generally being studied all around the world by different practitioners as well as researchers. Nevertheless, it is very much unusual for the researchers to conduct study or work in same institution or organizations; hence, this makes it extremely hard for them to share their information for mutually leading to an integrated solution like the s-health concept. Since, the notion of s-health 
is very new, there is an increasing requirement for collaboration, cooperation and interaction among diverse actors including researchers, practitioners, governments, physicians, etc. for defining a common mutual ground from the beginning, and hence, preventing redundant redesigns as well as over-spending.

2) Security and privacy: Even when s-health approach can help in mitigating various health related concerns, but its capability to obtain unparalleled among of data can jeopardize the citizens' privacy. Protection of privacy and security of the infrastructure is an inevitable issue that research community is still trying to redress. Privacy protection and security is foremost in around every facet of human life. Nevertheless, in smart city context, it is even of greater importance and the reason is because the information that is obtained is very personal. From the data or information that is collected in a smart city, it is possible to get knowledge about the habits of citizens, their social status, other personal information, and even the information related to their religion. All these personal information variables are extremely delicate in nature, and when they are integrated with the health information of an individual, the outcome is even more sensitive. Hence, it infers a great difficulty and several challenges that are still required to be studied. Certain efforts have been conducted to explain the concept of the privacy of citizens and to offer possible solution to protect that privacy [10][8]. In addition, various attempts are contributed towards the privacy protection in heath, Trust- worthy Health and Wellness (THaW) is one of the representative projects in this context [7][13]. The concept of THaW aims to resolve diverse challenges and difficulties to offer trustworthy and reliable information systems for wellness and health. In a similar way, the Strategic Healthcare IT Advanced Research Projects on Security (SHARPS) is another project that aims in making improvements in the foundations, requirements, development, design, and deployment of security, as well as on privacy methods and tools that are used for m-health. Such projects are the research priorities and difficulties with them are daunting [14][15].

\section{Opportunities}

The perception of s-health is created on a ground that it can use the smart cities' infrastructure that in turn can open an array of opportunities or potentials for the expansion and growth of new applications identified and explored services that are associated with health. Some of the opportunities that s-health can offer include:

1) Data collection, presentation, and analysis: It can help in practical redesigning of data related to health, because certain information that seems irrelevant can be of utmost importance for healthcare services [16][17]. S-health makes it possible to collect data from healthy people and patients in real-time and can further be combined with the data or information of city. Main routes, signs, as well as records of every citizen could be seamlessly integrated or combined with the data that is derived using pervasive cameras, sensors, forecasts, and weather reports. Hence, proper use of all the data can then become the prime pillar and basis for the shealth applications [18].

2) Prevention as well as administration of critical incidents: $s$-Health expected to also provide the precise techniques for avoidance as well as proficient management of acute plus chronic ailments plus accidents. Comprehend- shealth expected to be helpful in identifying the circumstance that needs intervention such as falls, cardiovascular events, accidents, and can offer optimized and automated management of such incidents such as providing the guidance as well as notifications to the establishment (Example 5) [19][20]. In the event, when there is a small and not very acute event, the patient can easily be guide to reach immediately at bordering health provider or pharmacy by using s-health. On the other hand, during the life-threatening circumstances, the traffic information can also be used to guide and dispatch the ambulance so that it can reach patient as early possible. Comprehensive and proper data or information analysis can also offer various techniques for more efficient prevention of disease, early detection of chronic ailments and diseases, and even recognizing the new risks and threats related to health [1][21][22].

3) Effectiveness and environmental assessment: Patient expected monitor information also used for identification of non-optimally manage situations or non-responsive patients to offer them treatments as well as to provide them efficient and effective healthcare assistance. As an example, s-health systems can help in recognizing the patients with chronic diseases with major signs that are inconsistent to the medicines prescribed to them such as abnormal heart rate, blood pressures, blood glucose, etc. Example of such data can be combined with the location, status, and current actions of patient for the sake of reducing false positives and recognize bona fide actions that needs interference [23][24]. Such application may also impose substantial influence on the new interventions' assessment in case of clinical trials. S-Health systems can seamlessly integrate patient's medical records, long-term patient monitor system, and efficient assessment methodologies with the data provided by city sensors. Such integration act as an ideal setting for providing high-quality personalized medicine. Environmental conditions including pollution, temperature, humidity, etc. and daily routes and activities of patients can also be used in order their dosing at an unprecedented level of detail, while the capability of the system to routinely and actively measure each intervention's effectiveness [10][25].

4) Engaging patients and families in managing their health: In s-health approach, the citizens are substantially empowered as well as assisted in an efficient manner for participating actively in the management of their health. Shealth systems can make use of medical records data as well as important signs for providing optimal and best guidance for everyday activities, habits, and tasks within the city [15]. For 
example, an s-health application can also offer the heart patients and those with respiratory diseases, with a best route by preventing the areas with high level of pollution [9][26][27].

5) Improving policy decisions: s-Health systems can also enable health management of public. Laws and preferences can also be "modified" to evaluate every district or city, on the basis of the data that is resulting from health hazard, population, weather, environment, and accessible infrastructure. There are boundless or limitless potential that arise from mining such data for the sake of optimizing decision making related to public health.

6) Epidemic control: s-health data as well as methodologies also radically enhance the competence of the state in detection and control of epidemics. The vital signs, activities, and locations of citizens can be utilized for detection of possibly the new cases during an epidemic, effectively recognizing the fields of enhanced risk, and managing a ranging outbreak in an effective manner. Such methods also be implemented in the detection as well as organization of other widespread risks related to health such as radiation or pollution from an trade incident [28].

7) Cost saving: Identified sectors that are exploited previously may impose a substantial impact on reducing the cost of health care [29][30][31]. Such reduction of cost will also be included with simultaneous boost in the efficiency of scheme and enhancement in the provision of services. Timely [7][32], optimization of the prevention and management of disease can further lead to decrease in unnecessary visits to hospitals and the development of acute events due to poor management of the patients with chronic conditions. Additionally, reduction in the action time and effective public health management can also offer optimal outcomes while offering reduction in costs on a national scale [33][34][35][36].

\section{CONCLUSION}

The extensive ICT adoption in the cities' context led to the evolution of smart cities. In a similar manner, using mobile technologies and ICT for issues related to health will led to appearance of monitoring of patient and health care in a ubiquitous way using e-health and $\mathrm{m}$-health. Whilst researchers are already focusing on the further development of the ideology of m-health plus smart cities, it can further believed that there is an increasing requirement for a new concept that can be known as smart health (s-health), which emerges by amalgamating the identified smart cities with mobile and electronic health services.

Upon introducing this new ideology of s-health plus by providing clarification of the scope of this concept, the research is trying to pave the way for prospect explore to have a clarified plus better concentration as well as a common explanation to enhance healthcare. The given editorial provided overview of the s-health concept, and help in analyzing most of the research fields related to it. The article also helped in discussing the major challenges that are generally faced during the development and implementation of s-health, and it also focuses on highlighting all the opportunities possible to implement the concept and the future potentials of s-health, which according to the researchers are boundless.

\section{FUNDING}

This research is funded by the Deanship of Scientific Research and Graduate Studies in Yarmouk University, Jordan.

\section{REFERENCES}

[1] Mamra and A. Mamra, "A Proposed Framework to Investigate the User Acceptance of Personal Health Records in A Proposed Framework to Investigate the User Acceptance of Personal Health Records in Malaysia using UTAUT2 and PMT," Int. J. Adv. Comput. Sci. Appl., no. March, 2017.

[2] M. B. Alazzam, A. B. D. Samad, H. Basari, and A. Samad, "PILOT STUDY OF EHRS ACCEPTANCE IN JORDAN HOSPITALS BY UTAUT2," vol. 85, no. 3, 2016.

[3] M. B. Alazzam, A. Samad, H. Basari, and A. S. Sibghatullah, "Trust in stored data in EHRs acceptance of medical staff : using UTAUT2," vol. 11, no. 4, pp. 2737-2748, 2016.

[4] M. B. Alazzam, Y. M. Al-sharo, and M. K. Al-, "DEVELOPING ( UTAUT 2 ) MODEL OF ADOPTION MOBILE HEALTH APPLICATION IN JORDAN E- GOVERNMENT," vol. 96, no. 12, 2018.

[5] M. B. Alazzam, "Physicians' Acceptance of Electronic Health Records Exchange: An Extension of the with UTAUT2 Model Institutional Trust," Adv. Sci. Lett., vol. 21, pp. 3248-3252, Feb. 2015.

[6] A. S. MB.Alazzam, "Review of Studies With Utaut As Conceptual Framework,” Eur. Sci. J., vol. 10, no. 3, pp. 249-258, 2015.

[7] M. R. Ramli, Z. A. Abas, M. I. Desa, Z. Z. Abidin, and M. B. Alazzam, "Enhanced convergence of Bat Algorithm based on dimensional and inertia weight factor,’ J. King Saud Univ. - Comput. Inf. Sci., 2018.

[8] M. Rasmi, M. B. Alazzam, M. K. Alsmadi, A. Ibrahim, R. A. Alkhasawneh, and S. Alsmadi, "Healthcare professionals acceptance Electronic Health Records system: Critical literature review ( Jordan case study ) Healthcare professionals ' acceptance Electronic Health Records system : Critical literature review ( Jordan case study ), ' Int. J. Healthc. Manag., vol. 0, no. 0, pp. 1-13, 2018.

[9] A. Mamra et al., "Theories and factors applied in investigating the user acceptance towards personal health records : Review study Theories and factors applied in investigating the user acceptance towards personal health records : Review study," Int. J. Healthc. Manag., vol. 0, no. 0, pp. $1-8,2017$.

[10] S. M.Alazzam, BASARI, "EHRs Acceptance in Jordan Hospitals By UTAUT2 Model: Preliminary Result," J. Theor. Appl. Inf. Technol., vol. 3178, no. 3, pp. 473-482, 2015.

[11] M. Doheir, B. Hussin, A. Samad, H. Basari, and M. B. Alazzam, "Structural Design of Secure Transmission Module for Protecting Patient Data in Cloud-Based Healthcare Environment," Middle-East J. Sci. Res., vol. 23, no. 12, pp. 2961-2967, 2015.

[12] Y. Mohammad Al-Sharo, G. Shakah, M. Sh Alkhaswneh, B. Zeyad Alju-Naeidi, and M. Bader Alazzam, "Classification of big data: machine learning problems and challenges in network intrusion prediction,” Int. J. Eng. Technol., vol. 7, no. 4, pp. 3865-3869, 2018.

[13] J. L. Fernández-Alemán, I. C. Señor, P. Á. O. Lozoya, and A. Toval, "Security and privacy in electronic health records: a systematic literature review.," J. Biomed. Inform., vol. 46, no. 3, pp. 541-62, Jun. 2013.

[14] R. G. Hollands, "Critical interventions into the corporate smart city," Cambridge J. Reg. Econ. Soc., vol. 8, no. 1, pp. 61-77, 2015.

[15] V. Inukollu, S. Arsi, and S. Ravuri, "Security Issues Associated With Big Data in Cloud Computing," Int. J. Netw. Secur. Its Appl., vol. 6, no. 3, pp. 45-56, 2014. 
[16] T. Nam and T. A. Pardo, "Conceptualizing smart city with dimensions of technology, people, and institutions," Proc. 12th Annu. Int. Digit. Gov. Res. Conf. Digit. Gov. Innov. Challenging Times - dg.o '11, p. 282, 2011.

[17] D. M. Mendez, I. Papapanagiotou, and B. Yang, "Internet of Things: Survey on Security and Privacy," Inf. Secur. J. A Glob. Perspect., vol. 00, no. 00, pp. 1-21, 2017.

[18] A. Schaffers, Hans. Komninos, Nicos Pallot, Marc Trousse, Brigitt Nilsson, Michael. Oliveira, "Smart cities and the future internet: Towards cooperation frameworks for open innovation," Lect. Notes Comput. Sci. (including Subser. Lect. Notes Artif. Intell. Lect. Notes Bioinformatics), vol. 6656, pp. 431-446, 2011.

[19] V. Albino, U. Berardi, and R. M. Dangelico, "Smart Cities : Definitions, Dimensions, Performance, and Initiatives Smart Cities : Definitions, Dimensions, Performance, and Initiatives," vol. 22, no. 2017, pp. 3-21, 2015.

[20] M. Zineddine and I. Privacy, "automated healthcare information privacy and security : the uae context," vol. 2012, pp. 311-318, 2012.

[21] A Zanella, N. Bui, a Castellani, L. Vangelista, and M. Zorzi, "Internet of Things for Smart Cities," IEEE Internet Things J., vol. 1, no. 1, pp. 22-32, 2014.

[22] C. D. Huang, R. S. Behara, and J. Goo, "Optimal information security investment in a Healthcare Information Exchange: An economic analysis," Decis. Support Syst., vol. 61, pp. 1-11, Nov. 2013.

[23] J. Singh, "Big Data: Tools and Technologies in Big Data," Int. J. Comput. Appl., vol. 112, no. 15, pp. 975-8887, 2015.

[24] D. Box and D. Pottas, "Improving Information Security Behaviour in the Healthcare Context," Procedia Technol., vol. 9, pp. 1093-1103, Jan. 2013.

[25] K. Agbele, H. Nyongesa, and A. Adesina, "leT and Infonnation Security Perspectives in E-Health Systems," vol. 4, no. 1, pp. 17-22, 2010.

[26] A. Solanas et al., "Smart health: A context-aware health paradigm within smart cities," IEEE Commun. Mag., vol. 52, no. 8, pp. 74-81, 2014.
[27] K. Jammoul, H. Lee, and K. Lane, "UNDERSTANDING USERS , TRUST AND THE MODERATING INFLUENCE OF PRIVACY AND SECURITY CONCERNS FOR MOBILE BANKING: AN ELABORATION," vol. 2014, pp. 1-11, 2014.

[28] A. Gawlik, L. Köster, H. Mahmoodi, and M. Winandy, "Requirements for Integrating End-to-End Security into Large-Scale EHR Systems," pp. $1-12$.

[29] A. Glasmeier and S. Christopherson, "Thinking about smart cities," Cambridge J. Reg. Econ. Soc., vol. 8, no. 1, pp. 3-12, 2015.

[30] D. Li, J. Shan, Z. Shao, X. Zhou, and Y. Yao, "Geomatics for smart cities - concept, key techniques, and applications," Geo-Spatial Inf. Sci., vol. 16, no. 1, pp. 13-24, 2013.

[31] J. I. Fernando and L. L. Dawson, "The health information system security threat lifecycle: an informatics theory.," Int. J. Med. Inform., vol. 78, no. 12, pp. 815-26, Dec. 2009.

[32] I. Park, "Essays on information assurance: Examination of detrimental consequences of information security, privacy, and extreme event concerns on individual and organizational use of systems," ProQuest LLC, 2010.

[33] L. Anthopoulos, M. Janssen, and V. Weerakkody, "A Unified Smart City Model (USCM) for Smart City Conceptualization and Benchmarking,” Int. J. Electron. Gov. Res., vol. 12, no. 2, pp. 77-93, 2016.

[34] J. Jin, J. Gubbi, S. Marusic, and M. Palaniswami, "An information framework for creating a smart city through internet of things," IEEE Internet Things J., vol. 1, no. 2, pp. 112-121, 2014.

[35] I. De, T. Díez, M. Lopez-coronado, and M. López-coronado, "Privacy and Security in Mobile Health Apps: A Review and Recommendations Privacy and Security in Mobile Health Apps: A Review and Recommendations," no. October 2017, 2014.

[36] M. Elkhodr, S. Shahrestani, and H. Cheung, "Enhancing the security of mobile health monitoring systems through trust negotiations," 2011 IEEE 36th Conf. Local Comput. Networks, pp. 754-757, Oct. 2011. 